\title{
"Accidents waiting to happen" - insights from a simple model on the emergence of infectious agents in new hosts.
}

\author{
Walter A. Boeger ${ }^{1}$, Sofia Feronato ${ }^{1}$, and Sabrina Araujo ${ }^{1}$ \\ ${ }^{1}$ Universidade Federal do Parana Setor de Ciencias Biologicas
}

May 4, 2021

\begin{abstract}
Summary: This study evaluates through modeling the possible individual and combined effect of three populational parameters of pathogens (reproduction rate; rate of novelty emergence; and propagule size) on the colonization of new host species putatively the most fundamental process leading to the emergence of new infectious diseases. The results are analyzed under the theoretical framework of the Stockholm Paradigm using IBM simulations to better understand the evolutionary dynamics of the pathogen population and the possible role of Ecological Fitting. The simulations suggest that all three parameters positively influence the success of colonization of new hosts by a novel parasite population but contrary to the prevailing belief, the rate of novelty emergence (e.g. mutations) is the least important factor. Maximization of all parameters result in a synergetic facilitation of the colonization and emulates the expected scenario for pathogenic microorganisms. The simulations also provide theoretical support for the retention of the capacity of fast-evolving lineages to retro-colonize their previous host species/lineage by ecological fitting. Capacity is, thus, much larger than we can anticipate. Hence, the results support the empirical observations that opportunity of encounter (i.e. the breakdown in mechanisms for ecological isolation) is a fundamental determinant to the emergence of new associations - especially Emergent Infectious Diseases - and the dynamics of host exploration, as observed in SARS-CoV-2. Insights on the dynamics of Emergent Infectious Diseases derived from the simulations and from the Stockholm Paradigm are discussed.
\end{abstract}

\section{Hosted file}

Revised no marks -Modelling the acquisition of new hosts.pdf available at https://authorea. com/users/391384/articles/520718--accidents-waiting-to-happen-insights-from-a-simplemodel-on-the-emergence-of-infectious-agents-in-new-hosts

\section{Hosted file}

fig1 (1).pdf available at https://authorea.com/users/391384/articles/520718--accidentswaiting-to-happen-insights-from-a-simple-model-on-the-emergence-of-infectious-agents-innew-hosts
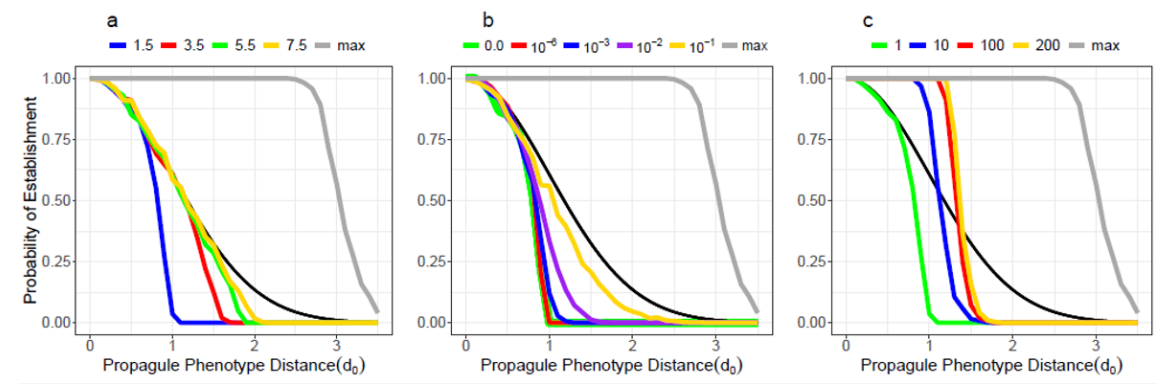

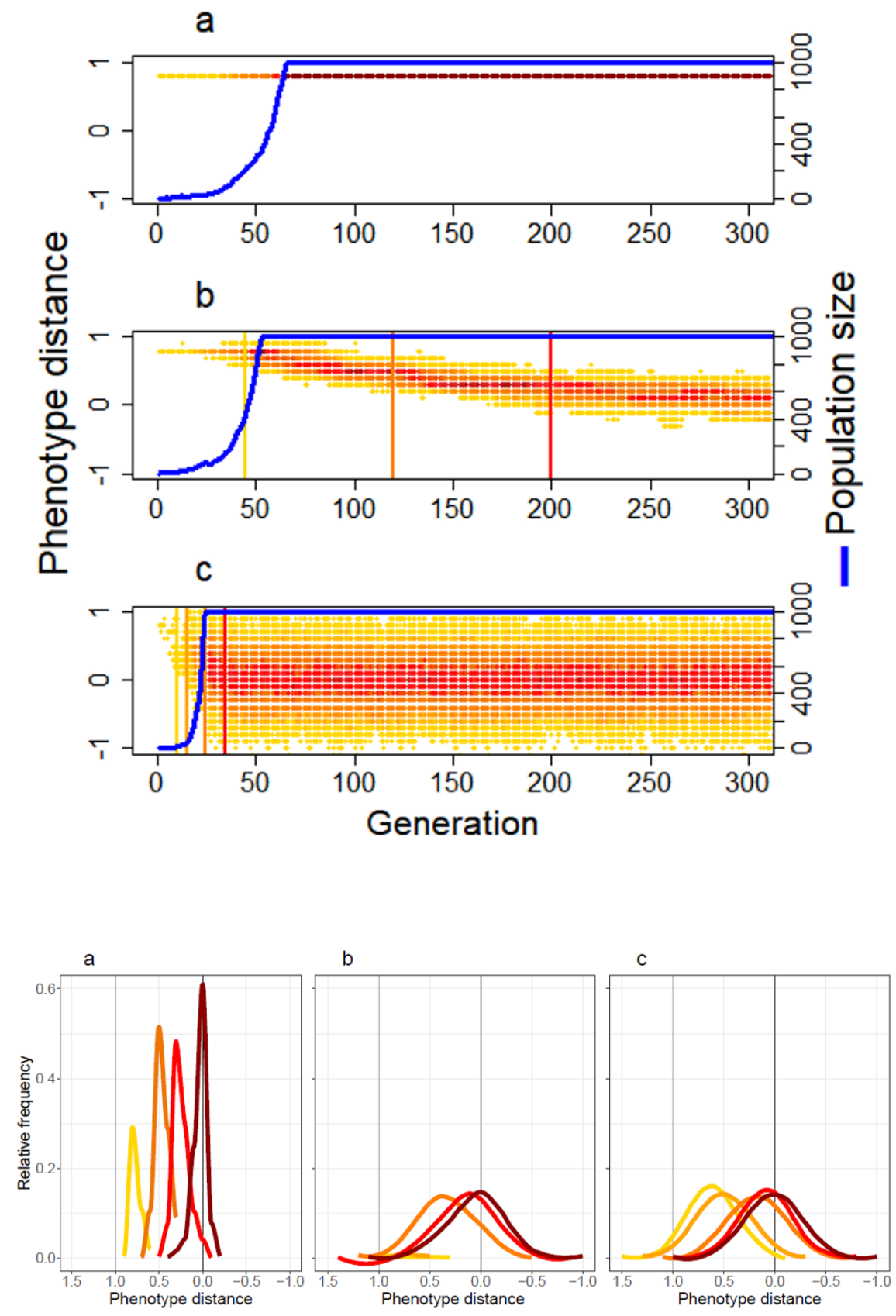